\title{
Chronic Radiodermatitis with Dystrophic Calcification Treated with Mohs Surgery
}

JOHN G. STONER, M.D.

NEIL A. SWANSON, M.D.

In 1941, Mohs ${ }^{1}$ first described a technique using microscopically controlled excisional surgery to treat basal- and squamous-cell carcinoma preceded by tissue fixation in situ. After trials with several fixatives, he settled on the use of a paste containing zinc chloride. ${ }^{2}$ In the majority of cases, Mohs surgery fixed-tissue technique has been supplanted by the fresh-tissue method. Removing tissue by this method has several advantages, including speed, greater conservation of healthy tissue, less pain and edema, and the possibility of immediate postoperative repair. Despite the many advantages of the fresh-tissue method, the classic fixed-tissue technique is still used in selected cases. Braun ${ }^{3}$ suggests that the fixed-tissue technique should be employed when cutaneous malignancies have invaded bone, deep structures, or organs like meninges and brain, when they are situated in narrow spaces like the auditory canals, involve highly vascular structures like the cavernous corpora of the penis, or when all that can be done is palliation of huge, unresectable malodorous neoplasms.

Whichever technique is employed, the indications for Mohs surgery have expanded greatly since the publication of Mohs' original paper. Any tumor amenable to frozen section interpretation can be treated, including such neoplasms as dermato-

John G. Stoner, M.D., Instructor, Department of Dermatology, and Neil A. Swanson, M.D., Assistant Professor, Departments of Dermatology and Otolaryngology, are with the University of Michigan Medical School, Ann Arbor, Michigan.

Address reprint requests to Neil A. Swanson, M.D., Department of Dermatology, C2065 Outpatient Building, University of Michigan, Ann Arbor, MI 48109. fibrosarcoma protuberans, ${ }^{4}$ adenocystic carcinoma of skin, malignant fibrohistiocytoma, leiomyosarcoma, hemangioendithelioma, verrucous carcinoma, meibomian gland carcinoma, and Bowen's disease. $^{5}$

Non-neoplastic indications include gangrene $e^{6,7}$ and chronic ulcerations of various etiology.$^{8}$ Ulceration is an infrequently described indication for Mohs surgery. This case illustrates the efficacy of the fixedtissue technique and reintroduces its use in the treatment of radiation-induced ulceration.

\section{CASE REPORT}

A 69-year-old white female presented with chronic pain, radiation dermatitis, and ulceration over the sacrum as a result of radiotherapy received 22 years previously for cervical carcinoma. Her initial examination 5 years previously revealed large deep ulcerations over the sacrum with a necrotic base. A biopsy taken from the ulcer margin demonstrated chronic radiodermatitis with dystrophic calcification. During the following year, these ulcers were managed conservatively by whirlpool soaks and surgical debridement. Debridement was quite painful despite local anesthetics, and she was lost to follow-up for the next 3 years. She reappeared in our clinic in early 1982 with a deep $4 \times 5.5 \mathrm{~cm}$ indurated ulcer over the left sacrum (Fig. 1). Repeat biopsy again showed chronic radiation dermatitis and scattered dystrophic calcification was seen on $x$-ray. At this time, it was elected to treat the ulcer by the Mohs surgery fixed-tissue technique. After debridement, zinc chloride paste was applied to the ulcer (Fig. 2) and the tissue was allowed to fix for 24 hours. The fixed tissue was then excised and examined histologically (Fig. 3). No foci of carci- 


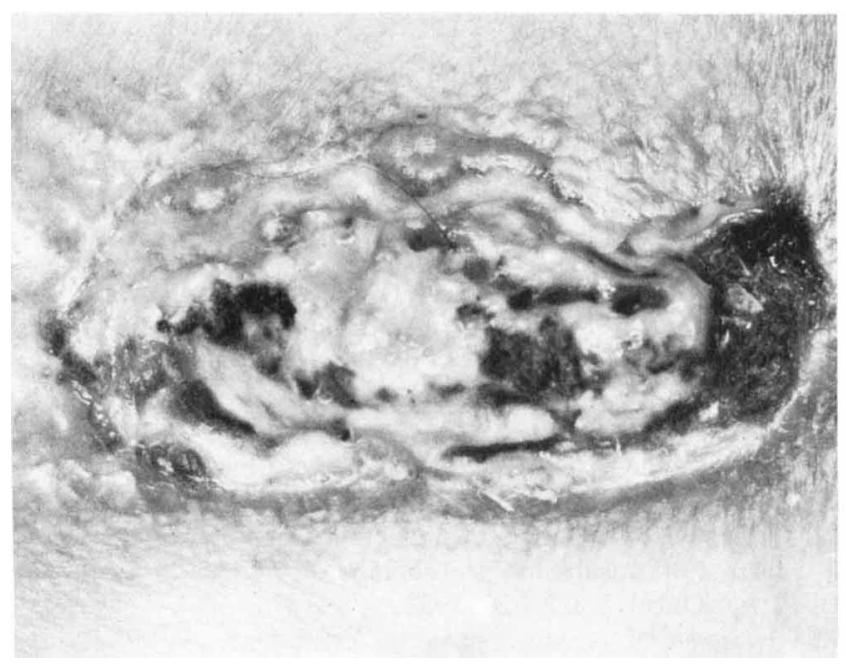

FIGURE 1. Indurated nonhealing ulcer over left sacrum.

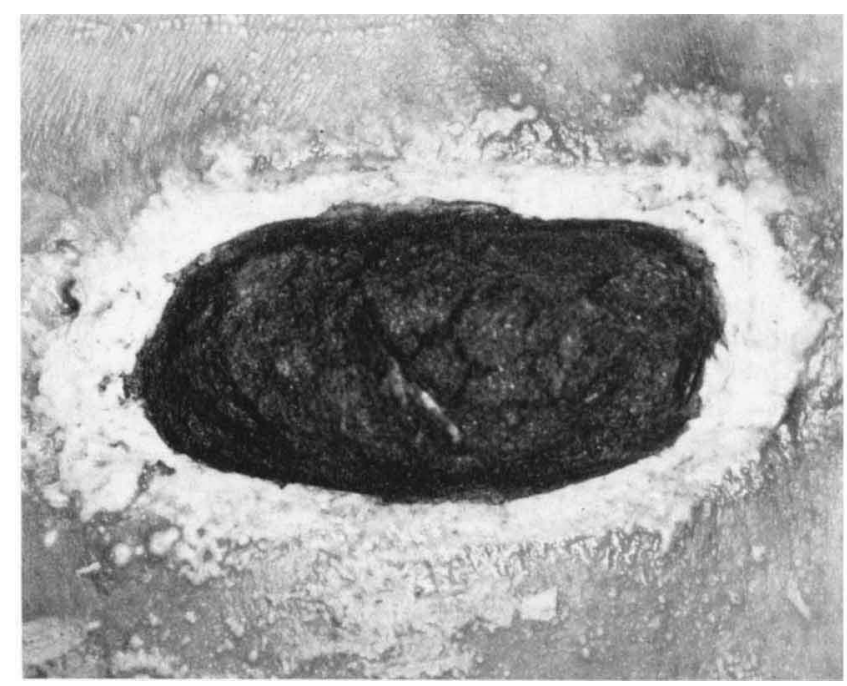

FIGURE 2. Ulcer fixed with zinc chloride paste.

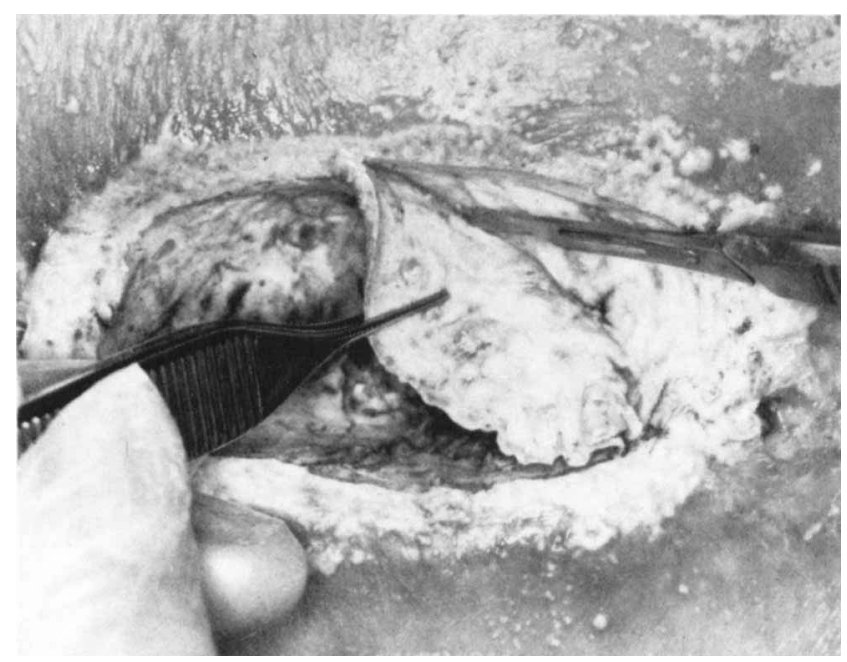

FIGURE 3. Fixed tissue is excised, mapped, and examined histologically.

1. Dermatol. Surg. Oncol. 11:2 February 1985

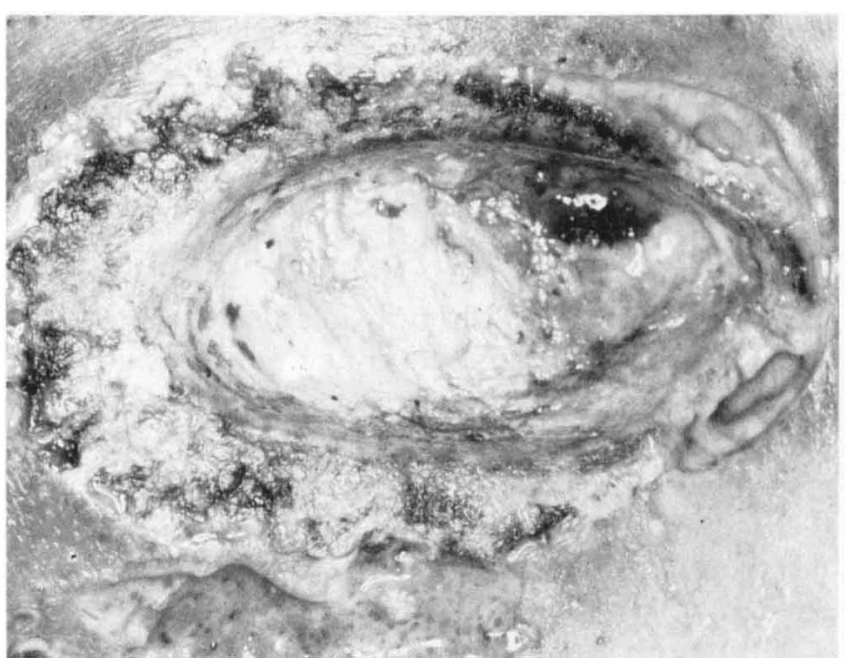

FIGURE 4. Immediate postoperative wound.

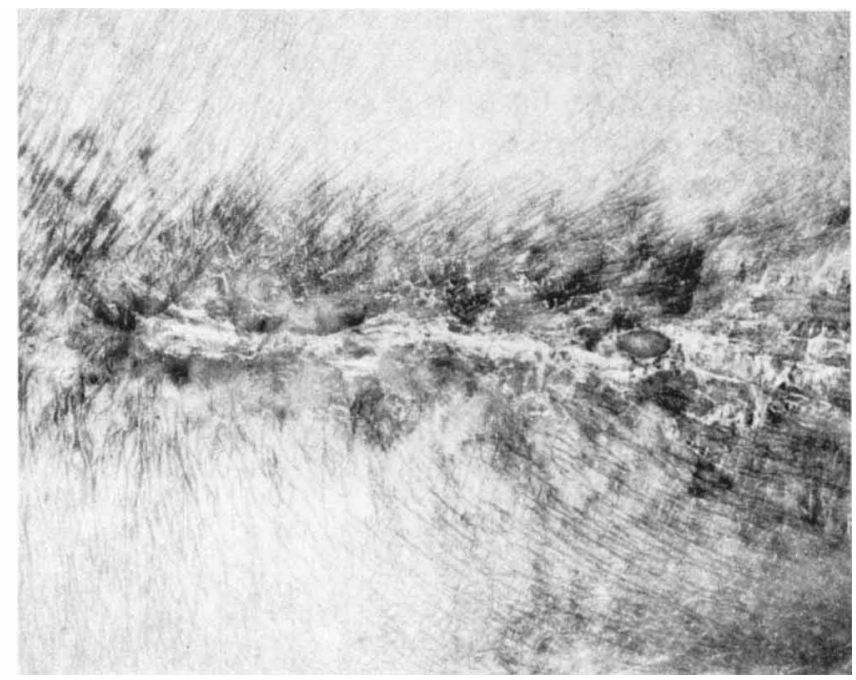

FIGURE 5. The wound has completely granulated 4 months postoperatively.

noma were observed. This process was repeated three times until all the calcified necrotic tissue was excised. The remaining fixed tissue was allowed to slough, leaving a hyperemic, clean wound with viable granulation tissue. After the final slough, the wound measured $6.5 \times 5$ centimeters and involved the full thickness of the paravertebral muscle but not underlying bone (Fig. 4). Now with a clean base and margins devoid of calcium, the wound granulated over the next 4 months (Fig. 5), leaving her with intact sacral skin for the first time in years.

\section{COMMENTS}

Dermal fibrosis and epidermal atrophy in radiationdamaged skin often results in cutaneous fragility, erosions, and ulcerations. Ulcers larger than $3 \mathrm{~cm}$ 
rarely heal spontaneously. ${ }^{9}$ Both conservative and surgical management often meet with poor results. The use of Mohs surgery fixed-tissue technique offers several advantages over these standard modes of surgery. First, fixation of the necrotic calcified tissue with zinc chloride paste allowed for easy mapping, excision, and histologic examination of the ulcer margins. Although in this case no carcinoma was found, it is well documented that both ionizing radiation ${ }^{10}$ and chronic ulceration ${ }^{11}$ predispose to the development of cutaneous carcinoma. Treatment of this ulcer by the fixed-tissue technique allowed for complete removal of the necrotic tissue and would have discovered carcinoma if it had been present.

Although the application of zinc chloride paste is somewhat painful, this discomfort was managed adequately with parenteral analgesics. However, once fixed, the tissue is rendered anesthetic, allowing debridement in a painless, bloodless field. Once a plane of healthy tissue was reached devoid of necrosis and calcium, the thin remaining layer of fixed tissue was allowed to slough. The exposed granulation tissue after this final slough is extremely vascular and highly resistant to infection and proliferates quickly. ${ }^{12}$ In our case, healing occurred in 4 months despite years of prior failure.

In conclusion, we suggest the addition of the Mohs surgery fixed-tissue technique to the treatment regimen for chronic radiation dermatitis with or without calcification to debride damaged tissue, facilitate healing, and obviate carcinoma.

\section{REFERENCES}

1. Mohs, F, E. Chemosurgery: A microscopically controlled method of cancer excision. Arch. Surg. 42:279-295, 1941.

2. Mohs, F. E., and Guyer, M. F. Pre-excisional fixation of tissue in the treatment of cancer in rats. Cancer Res., 1:49-51, 1941 .

3. Braun, M. The case for Mohs' surgery by the fixed tissut technique. J. Dermatol. Surg. Oncol. 7:634-640, 1981.

4. Peters, C. W., Hanke, C. W., et al. Chemosurgical reports: Dermatofibrosarcoma protuberans of the face. J. Dermatol. Surg. Oncol. 8:822-826, 1982

5. Swanson, N. A. Mohs surgery: Techniques, indications, applications, and future. Arch. Dermatol. 119:761-773, 1983.

6. Mohs, F. E., Severinghaus, E. L., and Schmidt, E. R. Conservative amputation of gangrenous parts by chemosurgery. Ann. Surg. 114:274-282, 1941.

7. Mohs, F. E. Chemosurgery: Microscopically Controlied Surgery for Skin Cancer. Springfield, Illinois, Charles C Thomas, 1978, pp. 279, 329, 331.

9. Strickland, P. Radiotherapy: Complications of radiotherapy, Br. J. Hosp. Med. 23:552, 556-560, 1980.

10. Traenkle, H. L. X-ray induced skin cancer in man. JNCI Monogr. 10:423-432, 1963.

11. Pennell, T. C., and Hightower, F. Malignant changes in postphlebetic ulcers. South. Med. J. 58:779-781, 1965.

12. Mohs, F. E. Chemosurgery: Microscopically Controlled Surgery for Skin Cancer. Springfield, Illinois, Charles C Thomas, 1978, pp. 21-22. 